\title{
Microspheres Assembled from Chitosan-Graft- Poly(lactic acid) Micelle-Like Core-Shell Nanospheres for Distinctly Controlled Release of Hydrophobic and Hydrophilic Biomolecules
}

Xufeng Niu, Zhongning Liu, Jiang Hu, Kunal J. Rambhia, Yubo Fan, Peter X. $\mathrm{Ma}^{*}$

To simultaneously control inflammation and facilitate dentin regeneration, a copolymeric micelle-in-microsphere platform is developed in this study, aiming to simultaneously release a hydrophobic drug to suppress inflammation and a hydrophilic biomolecule to enhance odontogenic differentiation of dental pulp stem cells in a distinctly controlled fashion. A series of chitosan-graft-poly(lactic acid) copolymers is synthesized with varying lactic acid and chitosan weight ratios, self-assembled into nanoscale micelle-like core-shell structures in an aqueous system, and subsequently crosslinked into microspheres through electrostatic interaction with sodium tripolyphosphate. A hydrophobic biomolecule either coumarin-6 or fluocinolone acetonide (FA) is encapsulated into the hydrophobic cores of the micelles, while a hydrophilic biomolecule either bovine serum albumin or bone morphogenetic protein 2 (BMP-2) is entrapped in the hydrophilic shells and the interspaces among the micelles. Both hydrophobic and hydrophilic biomolecules are delivered with distinct and tunable release patterns. Delivery of FA and BMP-2 simultaneously suppresses inflammation and enhances odontogenesis, resulting in significantly enhanced mineralized tissue regeneration. This result also demonstrates the potential for this novel delivery system to deliver multiple therapeutics and to achieve synergistic effects.

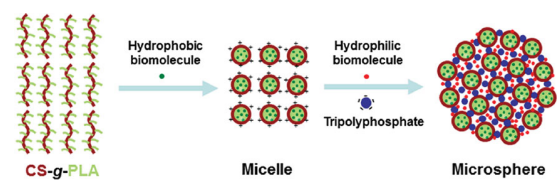

Dr. X. Niu, Dr. Z. Liu, Dr. J. Hu, Prof. P. X. Ma

Department of Biologic and Materials Sciences

University of Michigan

Ann Arbor, MI 48109, USA

E-mail: mapx@umich.edu

Dr. X. Niu, Prof. Y. Fan

Key Laboratory for Biomechanics and Mechanobiology of Ministry of Education

School of Biological Science and Medical Engineering

Beihang University

Beijing 100191, P. R. China

Dr. Z. Liu

Department of Prosthodontics

Peking University School and Hospital of Stomatology

Beijing 100081, P. R. China
K. J. Rambhia, Prof. P. X. Ma

Department of Biomedical Engineering

University of Michigan

Ann Arbor, MI 48109, USA

Prof. P. X. Ma

Macromolecular Science and Engineering Center

Department of Materials Science and Engineering

University of Michigan

Ann Arbor, MI 48109, USA 


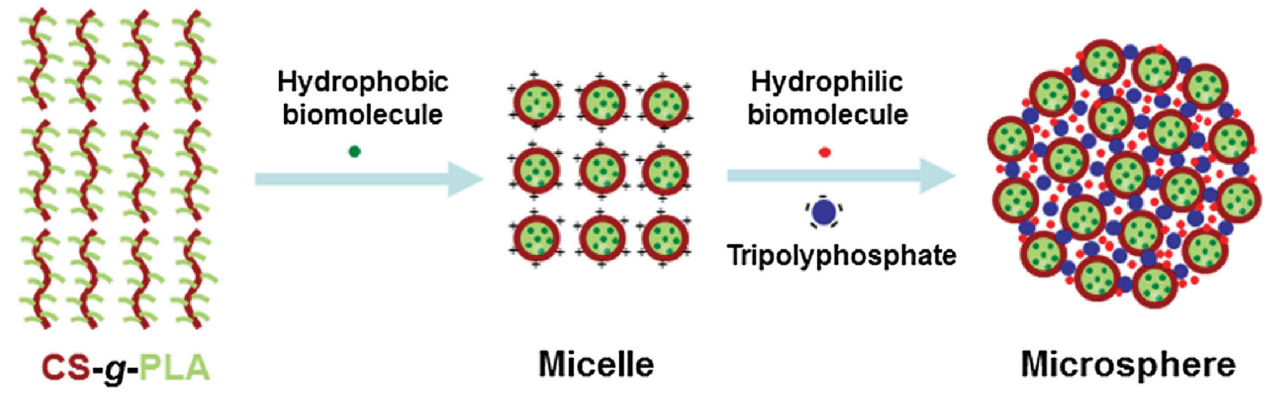

Figure 1. Schematic illustration for the preparation of CS-g-PLA copolymer based micelle-in-microsphere assemblies for the simultaneous co-delivery of hydrophobic and hydrophilic biomolecules. Hydrophobic biomolecules (green dots) were bound to hydrophobic cores of CS$g$-PLA micelle-like nanospheres. Hydrophilic biomolecules (red dots) were bound to hydrophilic shells and interspaces of CS- $g$-PLA micellelike nanospheres.

\section{Introduction}

The regeneration of tissues and organs is typically driven by the synergistic effects of various biomolecules acting on cells as they grow and mature. ${ }^{[1,2]}$ Therefore, simultaneous or sequential delivery of multiple biomolecules is typically needed in order to regenerate damaged, diseased, or previously removed tissue. ${ }^{[3,4]}$ Advanced co-delivery vehicles with dimensions ranging from micrometers to nanometers are being actively investigated. ${ }^{[5-8]}$ In comparison to single biomolecule delivery, co-delivery systems may possibly enhance therapeutic efficacy of tissue regeneration. ${ }^{[9,10]}$ However, biomolecules needed in tissue regeneration often have diverse biochemical and physiological properties. ${ }^{[11,12]}$

For dentin regeneration, drug delivery systems must control dental pulp inflammation and form a reparative dentin barrier to seal the pulp from exposure. Excessive or prolonged inflammation can impede tissue formation and remodeling, leading to tissue necrosis. ${ }^{[13]}$ When lipopolysaccharide (LPS) was used to induce an inflammatory dental microenvironment, odontoblastic differentiation and dentin regeneration were inhibited through the activation of the inhibitory NF- $\mathrm{KB}$ pathway. ${ }^{[14]}$ It was shown that sustained inflammation compromises bone morphogenetic protein (BMP)-induced bone formation. ${ }^{[15]}$ Therefore, in a clinical setting, local delivery of both an anti-inflammatory drug and an odontogenic growth factor (such as BMP) is necessary to generate a suitable microenvironment to rapidly regenerate dentin tissues to seal the defect and protect the pulp tissue.

As most first-line anti-inflammatory drugs are hydrophobic and potent odontogenic growth factors are hydrophilic, it remains a great challenge to co-deliver these two types of therapeutics. Fluocinolone acetonide (FA) is a steroid drug that has been widely used clinically to relieve inflammation in many inflammatory diseases including mucosal disorders and pulpitis. ${ }^{[14,16]}$ FA is hydrophobic and is only soluble in organic solvents such as dimethyl sulfoxide. ${ }^{[17]}$ On the contrary, BMP-2, an FDA-approved drug used to stimulate bone growth, is hydrophilic and its direct contact with an organic solvent during the encapsulation process compromises its bioactivity. ${ }^{[18,19]}$ As a result, most delivery systems are not optimal for codelivery of these two types of therapeutics.

For the delivery of single biomolecules, microspheres are advantageous because they can deliver a large payload and achieve long-term controlled release. ${ }^{[20-22]}$ Traditionally, single-component microspheres can be optimized for delivering one type of biomolecule or multiple biomolecules that have similar properties. ${ }^{[23-26]}$ Without matching biochemical properties, it is challenging to effectively encapsulate and preserve bioactivity of different biomolecules. ${ }^{[27,28]}$ Thus, a novel approach is needed to deliver both FA and BMP-2 for dentin regeneration.

In this work, we synthesized chitosan-graft-poly(lactic acid) (CS-g-PLA) copolymers and developed micelle-inmicrosphere assemblies (Figure 1), and used them to simultaneously co-deliver hydrophobic and hydrophilic biomolecules with distinct release profiles. This delivery system provides an innovative solution to the need for codelivery of biochemically distinct molecules to promote dentin regeneration in an inflammatory environment.

\section{Results and Discussion}

We first prepared CS-g-PLA copolymers with various amounts of poly(lactic acid) (PLA) by graft copolymerization. As shown in Figure 2A, we developed a new way to accomplish the desired copolymerization. Lactic acid (LA) was used to dissolve chitosan (CS) since the latter can only be dissolved in a weak acidic environment. Fourier transform infrared spectroscopy (FT-IR; Figure 2B) and ${ }^{1} \mathrm{H}$ nuclear magnetic resonance $\left({ }^{1} \mathrm{H}\right.$ NMR; Figure $2 \mathrm{C}$ ) characterizations were used to verify the success of this method of graft copolymerization. The characteristic peaks of high molecular weight PLA and their intensities increased accordingly when the weight ratios of LA/CS progressed from 10:1 to 40:1. The introduction of PLA grafts did not compromise the superior thermostability of CS (Figure S1, 
A

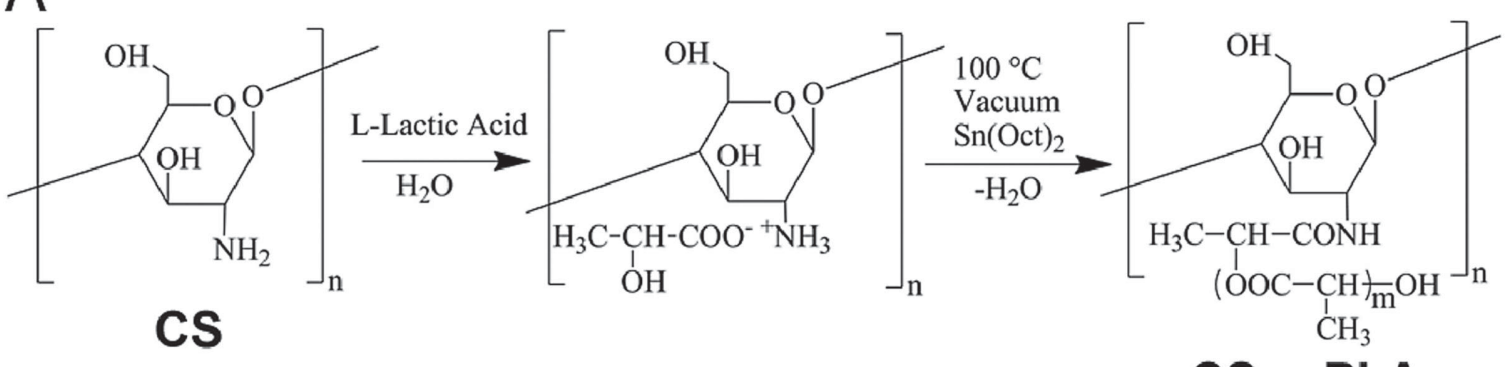

B

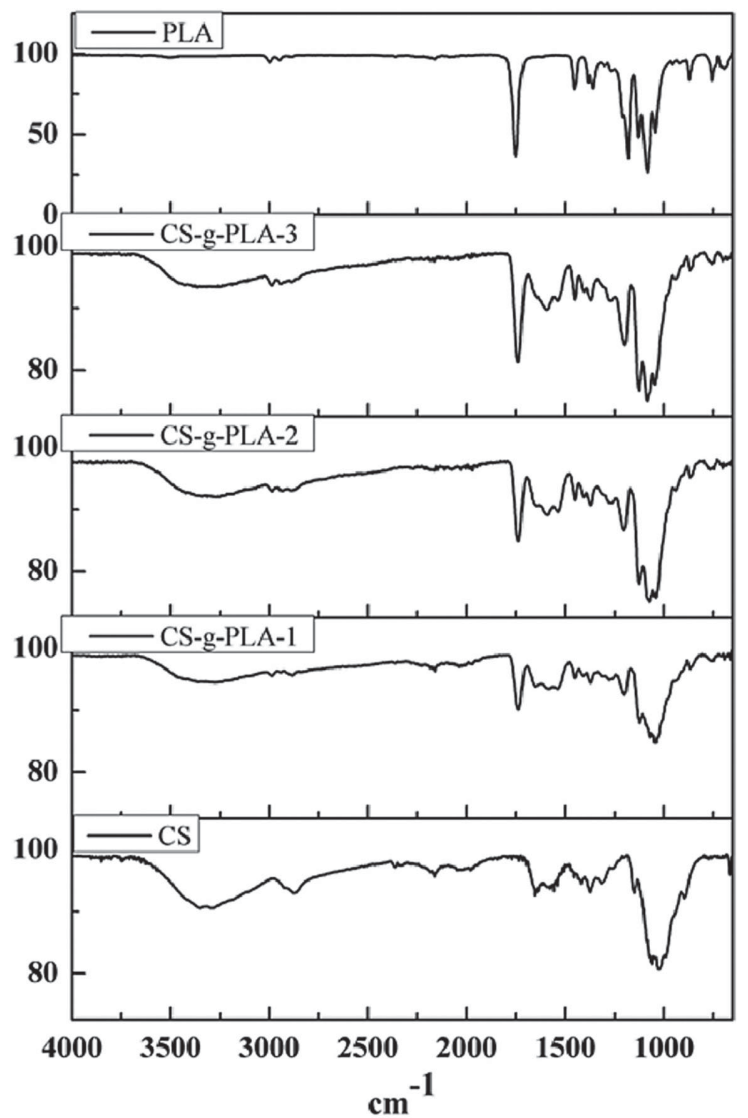

CS-g-PLA

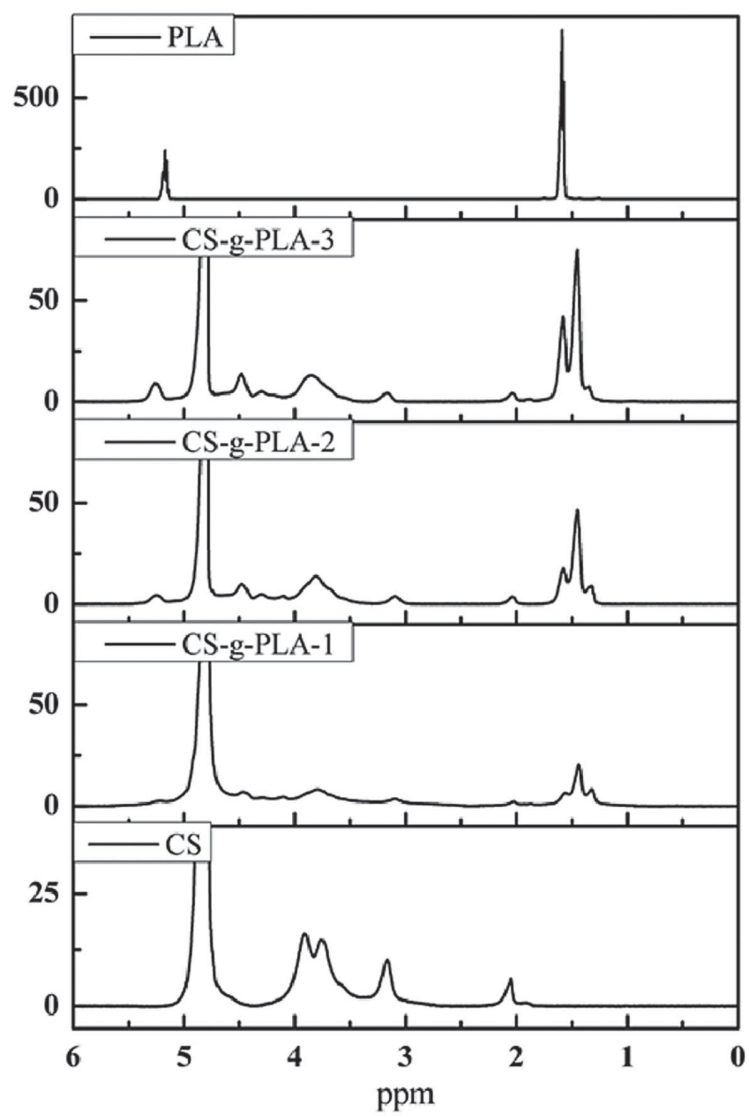

Figure 2. Synthesis and characterization of CS- $g$-PLA copolymers. A) Synthetic strategy of CS- $g$-PLA copolymers initiated by Sn(Oct) 2 . B) FT-IR spectral variations of CS- $g$-PLA copolymers with increasing LA/CS ratios of 10:1, 20:1, and 40:1, named as CS- $g$-PLA-1, 2, and 3, respectively. The spectra were compared with control CS and high molecular weight PLA. The representative signals of PLA appeared in all three CS- $g$-PLA copolymers, predominantly with ester carbonyl $(C=0)$ stretch at $1749 \mathrm{~cm}^{-1}$. C) ${ }^{1} \mathrm{H}$ NMR spectra variations of CS-g-PLA-1, 2, and 3, compared with the control CS and high molecular weight PLA. The spectra of all three CS- $g$-PLA copolymers were recorded in $\mathrm{D}_{2} \mathrm{O}$, while the spectra of $\mathrm{CS}$ and PLA were recorded in $\mathrm{DCl} / \mathrm{D}_{2} \mathrm{O}$ and $\mathrm{CDCl}$, respectively. The representative signals of PLA appeared in all three CS- $g$-PLA copolymers, predominantly with chemical shifts of methyl $\left(\mathrm{CH}_{3}\right)$ at $1.59 \mathrm{ppm}$ and methine $(\mathrm{CH})$ at $5.17 \mathrm{ppm}$. The grafting percentage (GP) of PLA was determined by the relative integral intensities of PLA and CS units as follows: GP $=\left(I_{5.17 \mathrm{ppm}} \times \mathrm{MW}_{\mathrm{L}-\mathrm{LA}}\right) /\left(I_{3.17} \mathrm{ppm} \times \mathrm{MW}\right.$ CS unit $) \times 100 \%$. Here, $\mathrm{MW}_{\mathrm{L}-\mathrm{LA}}$ is $90 \mathrm{~g} \mathrm{~mol}^{-1}$ and $\mathrm{MW}_{\mathrm{CS}}$ unit is $169.4 \mathrm{~g} \mathrm{~mol}^{-1}$ when its degree of deacetylation is $80 \%$. The calculated GP in CS- $g$-PLA-1, 2, and 3 was $26.0 \%, 61.1 \%$, and $120.6 \%$, respectively.

Supporting Information), which is beneficial for its clinical applicability as carrier materials.

To verify the formation of micelle-like particles of three synthesized CS-g-PLA copolymers, their structures were examined using transmission electron microscopy
(TEM) and a particle analyzer. TEM observation showed that both CS- $g$-PLA-2 and CS- $g$-PLA-3 could form spherical structures with diameters of $\approx 200 \mathrm{~nm}$ (Figure $3 \mathrm{~A}, \mathrm{C}$ ), whereas CS-g-PLA-1 failed to form an integrated structure (Figure S2, Supporting Information). Particle analysis

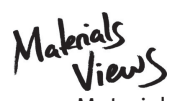

www.MaterialsViews.com 

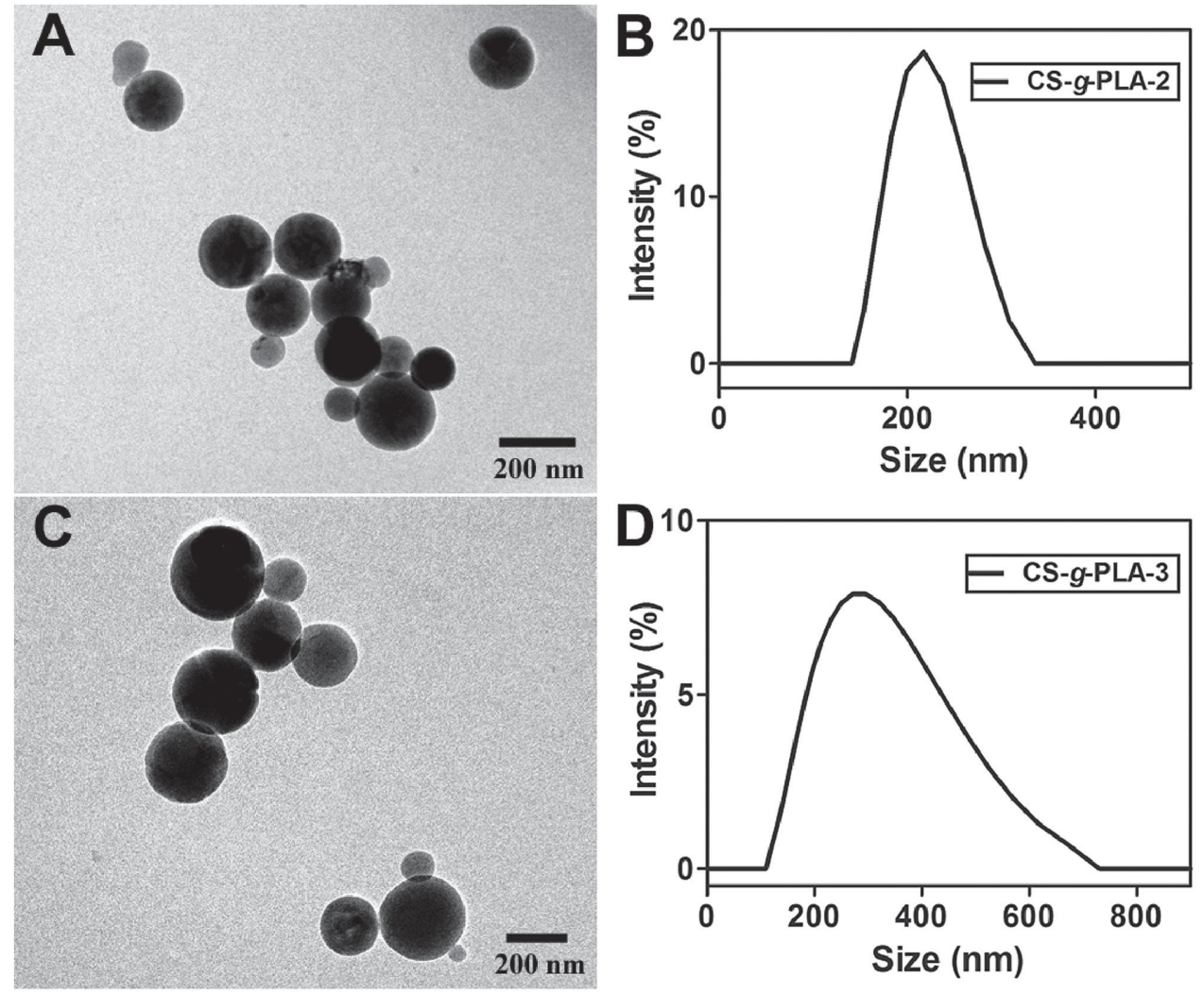

Figure 3. Characterization of CS- $g$-PLA copolymeric micelle-like nanospheres. A) TEM micrograph of CS-g-PLA-2 micelle-like nanospheres stained with phosphotungstic acid (PTA). B) Size distribution of CS- $g$-PLA-2 micelle-like nanospheres determined by particle analyzer. C) TEM micrograph of CS-g-PLA-3 micelle-like nanospheres stained with PTA. D) Size distribution of CS-g-PLA-3 micelle-like nanospheres determined by particle analyzer.

further revealed that the mean diameters of CS- $g$-PLA-2 and CS-g-PLA-3 micelles were 196.8 and $261.9 \mathrm{~nm}$ (Figure 3B,D), respectively, and the corresponding zeta potentials were 35.3 and $30.0 \mathrm{mV}$ (Figure S3, Supporting Information). The positive zeta potentials suggest that CS mainly constituted the shells while PLA, which is known to have a negative zeta potential, is primarily located in the cores. With increased LA/CS weight ratios, the cores became thicker accordingly and the micelles also increased in size.

Micelle-like structures have enhanced permeability and drug retention. In an ideal system for the delivery of biomolecules for tissue regeneration, the encapsulated biomolecules are delivered to the cells and the carrier material does not enter the cells. ${ }^{[11,29,30]}$ However, phagocytosis of the CS-g-PLA micelle-like nanospheres was observed when they were co-cultured with human dental pulp stem cells (DPSCs). Upon entering the cells, the nanospheres aggregated primarily within cellular lysosomes after co-culturing for $1 \mathrm{~d}$ (Figure S4, Supporting Information). To prevent phagocytosis of the small micellelike nanospheres, the core-shell structure was further crosslinked into microspheres in the presence of tripolyphosphate (TPP). As shown in Figure 4, both CS-g-PLA-2 and CS-g-PLA-3 micelles could be assembled into microspheres. CS- $g$-PLA-1 was omitted from these experiments because it did not form a micelle-like structure in an aqueous system. At high magnification, nano-sized protuberances were observed on the microsphere surfaces (Figure 4C,G), which corresponded to the micelle-like structure shown in Figure 3. After being crosslinked, the average diameters of CS- $g$-PLA-2 and CS- $g$-PLA- 3 microspheres were 72.7 and $78.7 \mu \mathrm{m}$, respectively (Figure 4D,H), and could not be phagocytized by DPSCs (Figure S4, Supporting Information).

Traditionally, CS and PLA have been used independently as delivery vehicles for a single type of biomolecule. ${ }^{[27,31-33]}$ To extend their function to co-delivery of different types of biomolecules, considerable efforts were made to develop CS and PLA-based composites often by physical blending. ${ }^{[34-37]}$ This led to a number of CS/PLA hybrids, but the two components tended to distribute unevenly within the composite because their miscibility is poor, due to the lack of a common solvents. Copolymers synthesized using CS with a low molecular weight PLA or its precursor as a chemical modifier are heterogeneous and therefore exhibit irreproducible properties. ${ }^{[38,39]}$ As a result, most CS and PLA composites 

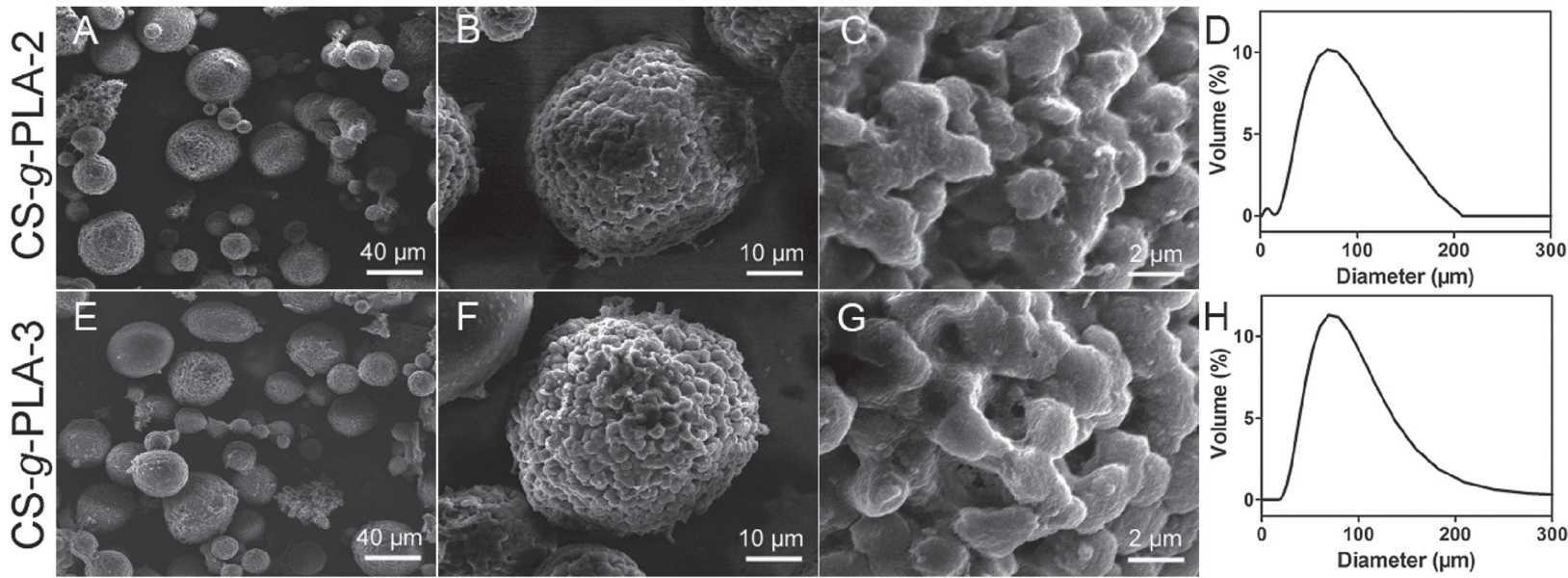

Figure 4. Morphology and size distributions of CS-g-PLA copolymeric microspheres assembled from micelle-like nanospheres. A-C) SEM micrographs of CS-g-PLA-2 microspheres with increasing magnification: A) 250x; B) 1000x;C) 5000x. D) The size distributions of CS-g-PLA-2 microspheres measure using a laser particle size analyzer. E-G) SEM micrographs of CS- $g$-PLA-3 microspheres with increasing magnification: E) 250x; F) 1000x; G) 5000x. H) The size distributions of CS-g-PLA-3 microspheres measured using a laser particle size analyzer.

are currently still unsuitable for co-delivery of multiple biomolecules.

In order to validate the potential of CS-g-PLA micellein-microsphere structure to co-deliver biomolecules with distinct physiochemical characteristics, coumarin-6 (a typical hydrophobic fluorochrome) and bovine serum albumin (BSA; a representative hydrophilic model protein) were sequentially encapsulated into CS-g-PLA
A Coumarin-6

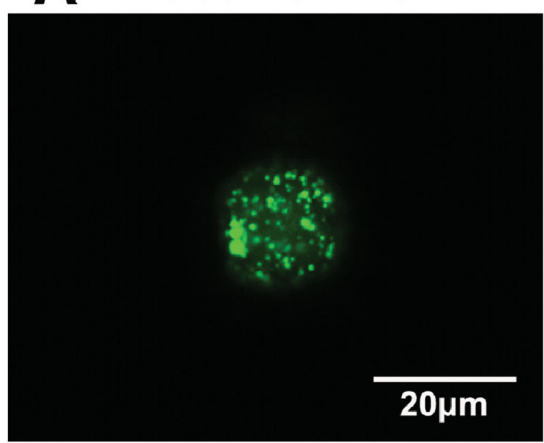

B

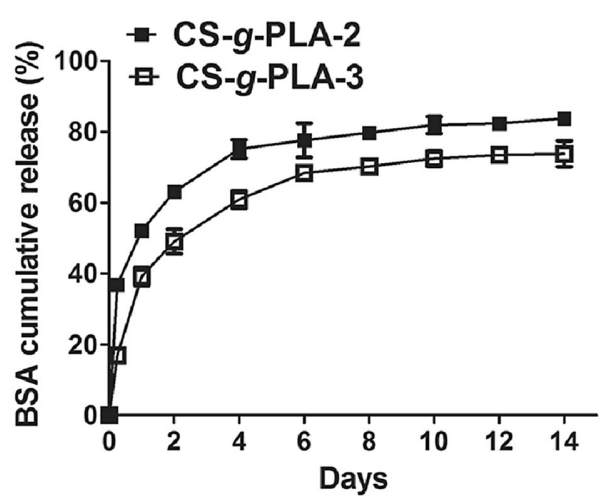

TRITC-BSA

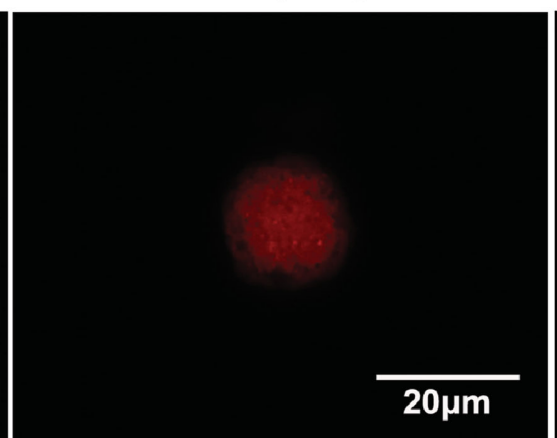

C

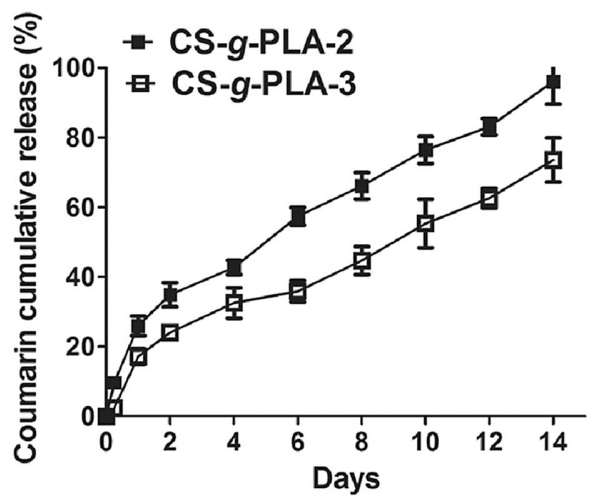

Figure 5. Distribution and co-delivery of hydrophobic coumarin- 6 and hydrophilic BSA (or TRITC-BSA) in CS- $g$-PLA copolymeric microspheres assembled from micelle-like nanospheres. A) Representative LSCM images of fluorescent coumarin- 6 (green fluorescence) and TRITC-BSA (red fluorescence) to exhibit their distinct distribution in microspheres. B) Release profiles of BSA from CS- $g$-PLA-2 and CS- $g$-PLA-3 microspheres in vitro in PBS buffer. A biphasic release pattern was presented over two weeks with a fast release phase in the first week and a sustained slow release phase in the second week. C) Release profiles of coumarin- 6 from CS- $g$-PLA- 2 and CS- $g$-PLA- 3 microspheres in vitro in PBS buffer. A nearly linear release pattern was observed during the first two weeks.

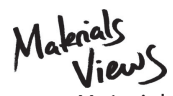

www.MaterialsViews.com 
microspheres and the in vitro release kinetics were investigated. Laser scanning confocal microscopy (LSCM) revealed that coumarin-6 (green fluorescence) and tetramethylrhodamine isothiocyanate (TRITC)-conjugated BSA (TRITC-BSA, red fluorescence) were observed in distinct areas of the microspheres (Figure 5A). Coumarin-6 appeared mainly within spherical areas and some further assembled aggregates, whereas TRITC-BSA was observed surrounding the spherical aggregates and formed a reticulate structure. During two weeks in phosphate buffered saline (PBS, pH 7.4), BSA displayed a biphasic release pattern characterized by a rapid release phase in the first week and a sustained slow release phase in the following week (Figure 5B). In contrast, coumarin-6 maintained a more linear release over the two week time period (Figure 5C). When compared with the CS-g-PLA-2 microspheres, the CS-g-PLA-3 microspheres showed decreased rates of BSA and coumarin- 6 release.

Both LSCM and in vitro release results further demonstrate that coumarin- 6 was located within the micellar cores while BSA was distributed on the surface of micellar shells and the interspaces among micelles. This is consistent with the schematic illustration in Figure 1 depicting the sequential addition of the two molecules during micelle and microsphere preparation. As a result, each molecule displayed a distinct in vitro release profile. Due to the hydrophilicity of CS, BSA released rapidly following immersion of microspheres in PBS. Because coumarin-6 was primarily entrapped in the cores of CS$g$-PLA micelles, its release correlated with the degradation of PLA and the diffusion through PLA. Because of the increased molecular weight and the amount of the PLA in the CS-g-PLA-3 microspheres, this preparation resulted in a slower release rate of coumarin-6. TPP crosslinking also had significant impact on the release of coumarin-6. In uncrosslinked CS-g-PLA micelle formulations, most coumarin- 6 was released within one week with slight burst release on the first day and a linear release for the following $6 \mathrm{~d}$ (Figure S5, Supporting Information). After assembling micelles into microspheres through crosslinking, the burst release was minimized and the release could be sustained over two weeks.

To further evaluate the utility of the new CS-g-PLA micelle-in-microsphere co-delivery system, hydrophobic FA and hydrophilic BMP-2 were loaded and delivered to treat DPSCs. In the CS- 9 -PLA-2 micelles, $5.8 \mathrm{mg} \mathrm{g}^{-1}$ of FA was loaded at a loading efficiency of $36.2 \%$. For BMP-2, $8.0 \mathrm{mg} \mathrm{g}^{-1}$ was loaded at $64.6 \%$ loading efficiency. (Table 1). As illustrated in Figure 1, hydrophobic FA molecules were encapsulated into hydrophobic cores of CS-g-PLA copolymeric micelle-like nanospheres. Hydrophilic BMP-2 molecules were then introduced and the micellar emulsion was crosslinked into microspheres through electrostatic interaction with TPP. Hydrophilic biomolecules were therefore mainly distributed in hydrophilic interspaces between the crosslinked micelle-like nanospheres and on the outer CS layer of micelles. BMP-2 was released at a faster rate to promote dentinogenesis at an early stage of treatment. FA was released in a sustained manner to inhibit inflammation during the entirety of the healing process. Paclitaxel and vascular endothelial growth factor (VEGF) were co-encapsulated in a microparticles/hydrogel composite previously, ${ }^{[40]}$ where VEGF was used to encourage the growth of endothelial cells while paclitaxel was used to inhibit the growth of smooth muscle cells. The major benefit of the present codelivery system is that different biomolecules can be presented compartmentally and their release profiles can be manipulated individually by varying LA and CS weight ratios (LA/CS) to meet the desired parameters for cell growth and tissue regeneration. In vitro release profiles (Figure 6A) showed similar patterns to coumarin-6 and BSA release (Figure 5B,C). After two weeks, the cumulative releases of both FA and BMP-2 reached $\approx 90 \%$. Despite a certain degree of deformation and conglutination, most microspheres still kept their original spherical shape after a two-week immersion in PBS (Figure S6, Supporting Information).

The effects of FA and BMP-2 released from CS- $g$-PLA-2 microspheres were investigated in LPS treated DPSCs. LPS is a major component of the outer membrane in gramnegative bacteria and can elicit inflammatory cytokine expression and apoptosis of DPSCs. In this cell culture model, LPS was used to induce an inflammatory reaction. ${ }^{[14]}$ Four groups were compared to a negative control: LPS alone, LPS and BMP-2, LPS and FA, and a co-delivery group of LPS with BMP-2 and FA. A constant linear release of FA was intended to persistently relieve the inflammation reaction. This approach was chosen because a large burst of FA levels has been reported to evoke unwanted

Table 1. FA and BMP-2 loading content and loading efficiency in CS-g-PLA-2 micelles and microspheres, respectively $(n=3)$.

\begin{tabular}{|c|c|c|c|c|c|c|}
\hline Sample & $\begin{array}{l}\text { FA dosage } \\
{\left[\mathrm{mg} \mathrm{g}^{-1}\right]}\end{array}$ & $\begin{array}{l}\text { FA loading content }{ }^{\mathrm{a})} \\
{\left[\mathrm{mg} \mathrm{g}^{-1}\right]}\end{array}$ & $\begin{array}{c}\text { FA loading efficiency } \\
{[\%]}\end{array}$ & $\begin{array}{c}\text { BMP-2 dosage } \\
{\left[\mathrm{mg} \mathrm{g}^{-1}\right]}\end{array}$ & $\begin{array}{c}\text { BMP-2 loading } \\
\text { content }^{\mathrm{b})} \\
{\left[\mathrm{mg} \mathrm{g}^{-1}\right]}\end{array}$ & $\begin{array}{l}\text { BMP-2 loading } \\
\text { efficiency }{ }^{b}[\%]\end{array}$ \\
\hline CS-g-PLA-2 & 10.0 & $5.8 \pm 1.3$ & $36.2 \pm 6.5$ & 10.0 & $8.0 \pm 1.4$ & $64.6 \pm 8.1$ \\
\hline
\end{tabular}

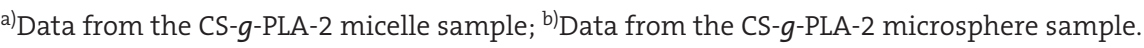



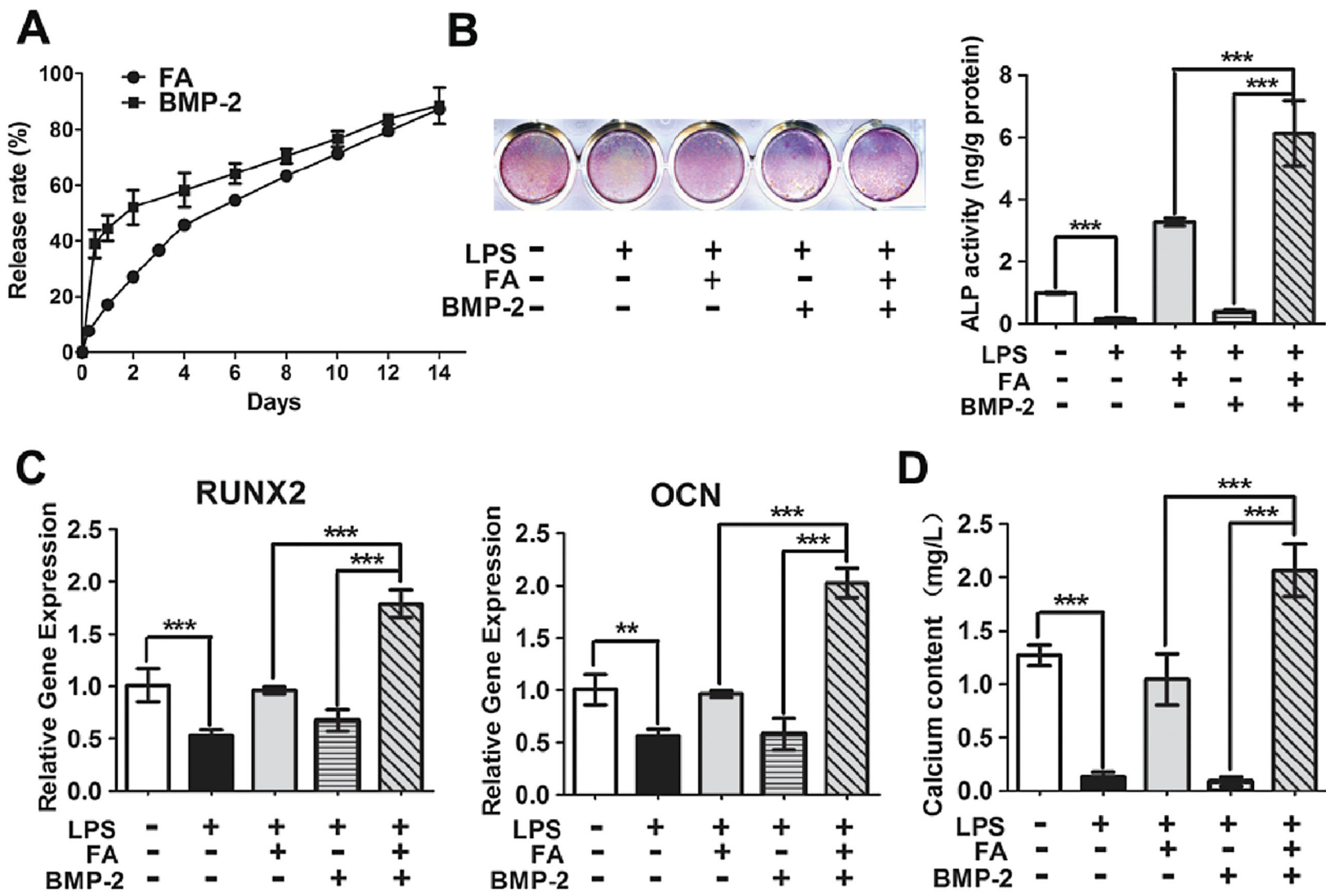

D

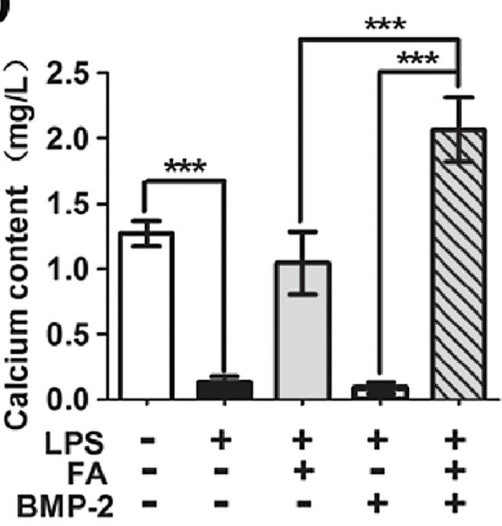

Figure 6. Synergistic effects of co-delivered FA and BMP-2 on mineralization of DPSCs under LPS treatment. A) Release profiles of FA and BMP-2 from CS- $g$-PLA-2 microspheres over two weeks in PBS buffer. B) ALP staining and activities of LPS-treated DPSCs after $7 \mathrm{~d}$ of incubation with CS- $g$-PLA-2 microspheres. C) RUNX2 and OCN gene expression profiles of LPS-treated DPSCs after $7 \mathrm{~d}$ of incubation with CS- $g$-PLA-2 microspheres. D) Calcium content quantification of LPS-treated DPSCs after $21 \mathrm{~d}$ of incubation with CS- $g$-PLA-2 microspheres.

side effects. ${ }^{[41]}$ Rapid BMP-2 release during the initial phase was designed to recruit DPSCs to the injury site and the subsequent sustained release of BMP-2 facilitated the differentiation of DPSCs into functional cells. Alkaline phosphatase (ALP), runt-related transcription factor 2 (RUNX2), and osteocalcin (OCN) are major biomarkers in hard tissue regeneration. Dentin sialophosphoprotein (DSPP), which is regarded as a dentin specific protein, is a major noncollagenous dentin matrix protein during dentin formation and is present at a high concentration in mature dentin. ${ }^{[42]}$ Therefore, ALP activity and gene expression of RUNX2, OCN, and DSPP were measured on day 7 of treatment. ALP activity was significantly decreased in DPSCs treated with LPS alone. Treatment with LPS and FA up-regulated ALP activity above negative control levels, suggesting FA successfully inhibited inflammation. Delivery of LPS and BMP-2 resulted in some recovery of ALP activity, but still below the levels of the negative control, whereas co-delivery of FA and BMP-2 synergistically enhanced the ALP activity when compared to single delivery groups (Figure $6 \mathrm{C}$ ). Consistently, RUNX2, OCN, and DSPP expression were down-regulated in LPS-treated DPSCs. Addition of FA recovered RUNX2,
OCN, and DSPP gene expression while BMP-2 alone had no effect. Co-delivery of FA and BMP-2 promoted the gene expression above negative control when compared with the single delivery groups (Figure 6D). To further investigate the effects of the co-delivery system for an extended period of time, calcium was then extracted and quantified on day 21 of treatment. Consistent with the ALP activity and the expression of odontogenic genes, the calcium content increased significantly with the co-delivery group, demonstrating the synergistic effect of FA and BMP-2 co-delivery (Figure 6B). In summary, CS-g-PLA-2 microspheres, by co-delivery of FA and BMP-2, can suppress inflammation effect and enhance dentin regeneration.

\section{Conclusions}

CS-g-PLA copolymeric microspheres assembled from micelle-like core-shell nanospheres have been developed as co-delivery vehicles of multiple biomolecules. This co-delivery system contains hydrophobic micellar cores and hydrophilic shells along with intermicellar spaces and has been demonstrated to effectively deliver

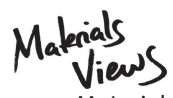

www.MaterialsViews.com 
water-insoluble anti-inflammatory drug FA and water-soluble osteo/odontogenic growth factor BMP-2, with distinct and tunable release patterns. This micelle-in-microsphere co-delivery platform has potential for dentin regeneration and other biomolecule-mediated tissue regeneration applications. This platform is particularly suitable for the concurrent delivery of synergistic hydrophobic and hydrophilic biomolecules.

\section{Experimental Section}

Preparation and Characterization of CS-g-PLA Copolymers: CS, L-lactic acid (L-LA), and Sn(Oct) ${ }_{2}$ were purchased from SigmaAldrich (St. Louis, MO, USA) and CS has an dynamic viscosity of $200-800 \mathrm{cP}$ and degree of deacetylation $75 \%-85 \%$. L-LA and CS with weight ratios of 10:1, 20:1, and 40:1 were co-dispersed in distilled water, respectively. The obtained transparent solutions were lyophilized to form CS lactate paste. Sn(Oct) (in tetrahydrofuran) with a molar ratio of $0.05 \%$ to the L-LA was added. Under rigorous mechanical stirring, the graft copolymerization was carried out at $100{ }^{\circ} \mathrm{C}$ in vacuum for $5 \mathrm{~h}$. The crude products were then extracted with excess amounts of toluene and further lyophilized. The obtained copolymers were named CS-g-PLA-1, CS-g-PLA-2, and CS-g-PLA-3 accordingly, and characterized by FT-IR (Perkin Elmer 1800), ${ }^{1} \mathrm{H}$ NMR (Varian MR400), differential scanning calorimetry (DSC, Perkin Elmer DSC-7), and thermogravimetric analysis (TGA, Perkin Elmer TGA-7).

Preparation and Characterization of CS-g-PLA Micelles: The micelles were prepared by dialysis method. The CS-g-PLA copolymers were dissolved in dimethyl sulfoxide (DMSO). The resulting solution was transferred to dialysis tubing (MWCO 6-8 kDa) and dialyzed against deionized water for $24 \mathrm{~h}$. The medium was renewed every $6 \mathrm{~h}$. The dialyzed solution was collected and characterized by TEM (JEOL JEM-2100F) and Particle Analyzer (Beckman Coulter Delsa Nano C). For TEM observation, the samples were dropped onto a carbon coated copper grid (200 mesh) and stained using PTA $(1 \%, v: v)$. The hydrophobic fluorochrome coumarin-6 or anti-inflammatory drug FA loaded micelles were prepared using the same method and these biomolecules were co-dissolved in DMSO with CS-g-PLA copolymers.

Aggregation of Micelles: The dialyzed solution was concentrated to $3 \%(\mathrm{w}: \mathrm{v})$ and used to fabricate microspheres by emulsion-ionic crosslinking technique. Typically, $10 \mathrm{~mL}$ micellar solution was dropped into $100 \mathrm{~mL}$ of mineral oil containing $2 \%$ (w:v) surfactant span-80 and agitated mechanically for $2 \mathrm{~h}$ at room temperature to form a water-in-oil (W/O) emulsion. Then, $12.5 \mathrm{~mL} 5 \%(\mathrm{w}: \mathrm{v})$ TPP solution was added dropwise to the emulsion and stirred for another $2 \mathrm{~h}$ to stabilize the microspheres through electrostatic interaction between CS- $g$-PLA micelles and TPP. The resulting microsphere suspension was repeatedly washed with excess amounts of petroleum ether and 2-propanol, and then lyophilized. The morphology of microspheres was observed under scanning electron microscope (SEM; Philips XL30 FEG). The mean size and size distribution of microspheres were evaluated by laser particle size analyzer (Mastersizer 2000). The dried microspheres were suspended in deionized water and sonicated for $1 \mathrm{~min}$ before measurement. The measuring ranges were $0.020-2000 \mu \mathrm{m}$. The microspheres loaded with hydrophilic BSA or BMP-2 were prepared using the same method and these biomolecules were dissolved in CS-g-PLA micellar solution. BSA was replaced by TRITC-BSA for LSCM characterization (Nikon TS-100).

In Vitro Release: The micellar aggregates encapsulated with various hydrophobic and hydrophilic biomolecules were immersed in $0.01 \mathrm{~m}$ sterile PBS (pH 7.4) and kept at $37^{\circ} \mathrm{C}$ on an orbital shaker. At predetermined time intervals, the supernatant was collected and equivalent volume of fresh PBS was added to each sample. The coumarin- 6 concentration in the release medium was determined using a Varioskan Flash multimode reader (Thermo Scientific) with excitation $450 \mathrm{~nm}$ and emission $505 \mathrm{~nm}$. The FA concentration was determined using a UV spectrometer with detection wavelength of $237 \mathrm{~nm}$. The BSA concentration was determined using Micro BCA protein assay kit (Thermo scientific, Rockford, IL, USA) according to the manufacturer's instructions. The BMP-2 concentration was analyzed using a commercially available human BMP-2 enzymelinked immunosorbent assay (ELISA) kit (PeproTech, Rocky Hill, NJ, USA) following the manufacturer's protocol.

Cell Culture and Seeding: Human DPSCs were obtained from the Center of Craniofacial Molecular Biology, School of Dentistry, University of Southern California, which were isolated according to the protocol reported previously. ${ }^{[43]}$ The DPSCs were cultured with alpha modification of Eagle's minimum essential medium ( $\alpha$-MEM, Invitrogen, Carlsbad, CA) supplemented with $10 \%$ fetal bovine serum (FBS, Gibico, USA) and penicillin-streptomycin (100 U mL ${ }^{-1}$ and $100 \mu \mathrm{gL}^{-1}$, Gibico, USA), and incubated at $37{ }^{\circ} \mathrm{C}$ in $5 \% \mathrm{CO}_{2}$. The culture medium was changed every two days. DPSCs of passages 4-6 were used in the following studies.

ALP Assay: ALP activity was examined using Sensolyte pNPP Alkaline Phosphatase Assay Kit (AnaSpec, Fremont, CA) according to the manufacturer's procedures. Briefly, DPSCs at a density of $2 \times 10^{4}$ cells per well were seeded in 24 -well plates. On the following day, cells were cultured in odontogenic medium, which contained $\alpha$-MEM supplemented with $10 \%$ FBS, $50 \mu \mathrm{gL}^{-1}$ ascorbic acid, and $5 \times 10^{-3} \mathrm{M} \beta$-glycerophosphate. They were then incubated on an orbital shaker at $37{ }^{\circ} \mathrm{C}$ in $5 \% \mathrm{CO}_{2}$. CS-g-PLA microspheres $\left(0.5 \mathrm{mg} \mathrm{mL}^{-1}\right)$ were added in odontogenic medium with $2.0 \mu \mathrm{g} \mathrm{mL}^{-1} \mathrm{LPS}$. Half of the medium was changed every two days. After DPSCs were cultured for $7 \mathrm{~d}$, the cells were rinsed with PBS and washing buffer, homogenized in a $250 \mu \mathrm{L}$ lysis buffer on ice for $10 \mathrm{~min}$. The samples with lysis buffer were centrifuged at $2500 \mathrm{~g}$ at $4{ }^{\circ} \mathrm{C}$ for $10 \mathrm{~min}$ and $50 \mu \mathrm{L}$ supernatant was used for ALP activity quantification assay. The absorbance was measured at $405 \mathrm{~nm}$ with Varioskan Flash multimode reader. The ALP activity of cells was normalized with total protein content that was quantified by Micro BCA protein assay kit. Meanwhile, ALP staining was further examined by using Leukocyte Alkaline Phosphatase Kit (Sigma-Aldrich, St. Louis, MO, USA) according to the manufacturer's protocols.

Quantitative Real Time Polymerase Chain Reaction: The DPSCs were cultured with CS- $g$-PLA microspheres for $7 \mathrm{~d}$ and they were treated consistently with the above ALP assay. Total RNA was extracted from DPSCs by using RNA Mini kit (Qiagen, Valencia, CA) and $2 \mu \mathrm{g}$ total RNA was converted to cDNA with reverse transcription reagents (Taqman, Applied Biosystems, 
Foster City, CA). Real time polymerase chain reaction (PCR) was carried out in a total volume of $30 \mu \mathrm{L}$ in Taqman Universal PCR Master Mix (Applied Biosystems) with $2 \mu \mathrm{L}$ cDNA and $1.5 \mu \mathrm{L}$ predesigned primers and probes (Applied Biosystems) for RUNX2 (Hs00231692_m1), OCN (Hs01587813_g1), and DSPP (Hs00171962_ml). The reactions were performed using ABI Prism 7500 Real time PCR System (Applied Biosystems). The gene expression was normalized against housekeeping gene glyceraldehyde-3-phosphate dehydrogenase (GAPDH) expression. Calcium Content Quantification: After $21 \mathrm{~d}$ of culture, CS-g-PLA microspheres treated DPSCs were rinsed two times with PBS, and $200 \mu \mathrm{L}$ of $0.5 \mathrm{~N}$ hydrochloric acid was added to each well. The lyses were incubated overnight on an orbital shaker and collected in tubes. The total calcium content was tested following instruction provided by the Stanbio Laboratory calcium liquicolor kit (Boerne, TX, USA).

Statistical Analysis: Data were expressed as mean \pm standard deviation (SD). One-way analysis of variance (ANOVA) was used to test the between-group differences. Statistical significance was determined as $P<0.05$.

\section{Supporting Information}

Supporting Information is available from the Wiley Online Library or from the author.

Acknowledgements: The authors gratefully acknowledge the financial support from the US National Institutes of Health (NIDCR DE022327 and DE015384, NHLBI HL114038: to P.X.M.), the US Department of Defense (W81XWH-12-2-0008: to P.X.M.), the National Natural Science Foundation of China (Grant Nos. 31470915 and 11272038: to X.N., and Grant No. 81400498: to Z.L.), and the China Scholarship Council (X.N.).

Received: January 15, 2016; Published online: March 14, 2016; DOI: $10.1002 / \mathrm{mabi} .201600020$

Keywords: chitosan; co-delivery; controlled release; microspheres; poly(lactic acid)

[1] P. X. Ma, Adv. Drug Delivery Rev. 2008, 60, 184.

[2] G. B. Wei, P. X. Ma, Adv. Funct. Mater. 2008, 18, 3568.

[3] D. H. Kempen, L. Lu, A. Heijink, T. E. Hefferan, L. B. Creemers, A. Maran, M. J. Yaszemski, W. J. Dhert, Biomaterials 2009, 30, 2816.

[4] C. M. Curtin, E. G. Tierney, K. McSorley, S. A. Cryan, G. P. Duffy, F. J. O’Brien, Adv. Healthcare Mater. 2015, 4, 223.

[5] J. Zhang, H. Sun, P. X. Ma, ACS Nano 2010, 4, 1049.

[6] L. L. Chen, T. Jiang, C. H. Cai, L. O. Wang, J. P. Lin, X. G. Cao, Adv. Healthcare Mater. 2014, 3, 1508.

[7] R. A. Perez, A. El-Fiqi, J. H. Park, T. H. Kim, J. H. Kim, H. W. Kim, Acta Biomater. 2014, 10, 520.

[8] J. Hu, P. X. Ma, Pharm. Res. 2011, 28, 1273.

[9] D. Liu, L. M. Bimbo, E. Makila, F. Villanova, M. Kaasalainen, B. Herranz-Blanco, C. M. Caramella, V. P. Lehto, J. Salonen, K. H. Herzig, J. Hirvonen, H. A. Santos, J. Controlled Release 2013, 170, 268.

[10] M. Khan, Z. Y. Ong, N. Wiradharma, A. B. E. Attia, Y. Y. Yang, Adv. Healthcare Mater. 2012, 1, 373.
[11] F. M. Chen, M. Zhang, Z. F. Wu, Biomaterials 2010, 31, 6279.

[12] D. H. Kempen, L. B. Creemers, J. Alblas, L. Lu, A. J. Verbout, M. J. Yaszemski, W. J. Dhert, Tissue Eng., Part B 2010, 16, 551.

[13] P. R. Cooper, M. J. Holder, A. J. Smith, J. Endodont. 2014, 40, S46.

[14] Z. N. Liu, T. Jiang, X. Z. Wang, Y. X. Wang, Brit. J. Pharmacol. 2013, 170, 1262.

[15] R. L. Huang, Y. Yuan, G. M. Zou, G. Liu, J. Tu, O. Li, Stem Cells Dev. 2014, 23, 277.

[16] P. Youngnak-Piboonratanakit, K. Dhanuthai, K. Thongprasom, P. Luckprom, W. Sarideechaigul, L. Luangjarmekorn, M. Azuma, J. Oral Pathol. Med. 2009, 38, 689.

[17] W. Muincharern, P. Louwakul, P. Pavasant, V. Lertchirakarn, J. Endodont. 2011, 37, 181.

[18] P. J. Shi, K. Chen, J. C. H. Goh, Adv. Healthcare Mater. 2013, 2, 934.

[19] S. S. S. Lee, E. L. Hsu, M. Mendoza, J. Ghodasra, M. S. Nickoli, A. Ashtekar, M. Polavarapu, J. Babu, R. M. Riaz, J. D. Nicolas, D. Nelson, S. Z. Hashmi, S. R. Kaltz, J. S. Earhart, B. R. Merk, J. S. Mckee, S. F. Bairstow, R. N. Shah, W. K. Hsu, S. I. Stupp, Adv. Healthcare Mater. 2015, 4, 131.

[20] V. R. Sinha, A. Trehan, J. Controlled Release 2003, 90, 261.

[21] X. Liu, X. Jin, P. X. Ma, Nat. Mater. 2011, 10, 398.

[22] J. H. Xu, H. Zhao, W. J. Lan, G. S. Luo, Adv. Healthcare Mater. 2012, 1, 106.

[23] C. Wischke, S. P. Schwendeman, Int. J. Pharm. 2008, 364, 298.

[24] X. Niu, O. Feng, M. Wang, X. Guo, O. Zheng, J. Controlled Release 2009, 134, 111.

[25] F. B. Basmanav, G. T. Kose, V. Hasirci, Biomaterials 2008, 29, 4195.

[26] H. O. Wang, X. H. Yan, G. L. Li, C. Pilz-Allen, H. Mohwald, D. Shchukin, Adv. Healthcare Mater. 2014, 3, 825.

[27] G. B. Wei, O. M. Jin, W. V. Giannobile, P. X. Ma, Biomaterials 2007, 28, 2087.

[28] Z. M. Wang, K. F. Wang, X. Lu, C. Li, L. Han, C. M. Xie, Y. L. Liu, S. X. Ou, G. Zhen, Adv. Healthcare Mater. 2015, 4, 927.

[29] Z. P. Zhang, J. Hu, P. X. Ma, Adv. Drug Delivery Rev. 2012, 64, 1129.

[30] T. N. Vo, F. K. Kasper, A. G. Mikos, Adv. Drug Delivery Rev. 2012, 64, 1292.

[31] X. Niu, O. Feng, M. Wang, X. Guo, O. Zheng, J. Microencapsulation 2009, 26, 297.

[32] S. Fusco, G. Chatzipirpiridis, K. M. Sivaraman, O. Ergeneman, B. J. Nelson, S. Pane, Adv. Healthcare Mater. 2013, 2, 1037.

[33] R. M. Rasal, A. V. Janorkar, D. E. Hirt, Prog. Polym. Sci. 2010, 35, 338.

[34] M. Prabaharan, M. A. Rodriguez-Perez, J. A. de Saja, J. F. Mano, J. Biomed. Mater. Res. B 2007, 81B, 427.

[35] Y. Wan, H. Wu, A. X. Yu, D. J. Wen, Biomacromolecules 2006, $7,1362$.

[36] N. Bhattarai, Z. S. Li, J. Gunn, M. Leung, A. Cooper, D. Edmondson, O. Veiseh, M. H. Chen, Y. Zhang, R. G. Ellenbogen, M. O. Zhang, Adv. Mater. 2009, 21, 2792.

[37] R. A. Pérez, J. E. Won, J. C. Knowles, H. W. Kim, Adv. Drug Delivery Rev. 2013, 65, 471.

[38] H. Feng, C. M. Dong, Biomacromolecules 2006, 7, 3069.

[39] X. Niu, L. Wang, P. Chen, X. Li, G. Zhou, O. Feng, Y. Fan, Macromol. Chem. Phys. 2013, 214, 700.

[40] L. Wei, J. Lin, C. Cai, Z. Fang, W. Fu, Eur. J. Pharm. Biopharm. 2011, 78, 346.

[41] H. Henneicke, S. J. Gasparini, T. C. Brennan-Speranza, H. Zhou, M. J. Seibel, Trends Endocrinol. Metab. 2014, 25, 197.

[42] M. Prasad, W. T. Butler, C. Oin, Connect. Tissue Res. 2010, 51, 404.

[43] S. Gronthos, M. Mankani, J. Brahim, P. G. Robey, S. Shi, Proc. Natl. Acad. Sci. USA 2000, 97, 13625.

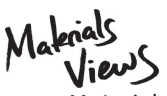

www.MaterialsViews.com 